\title{
Comparación entre las cargas planificadas y ejecutadas en el entrenamiento defútbol sala: la doble escala Comparison of planned and executed loads in futsal training: the double scale
}

\author{
Javier Álvarez Medina, Víctor Murillo Lorente \\ Universidad de Zaragoza (España)
}

Resumen. El objeto de este estudio es la relación de la percepción de la intensidad del entrenamiento de los jugadores con la carga planificada por el cuerpo técnico (doble escala). Participaron 12 jugadores profesionales de fútbol sala español durante toda una temporada de 40 semanas. Para la recogida de datos se utilizó la Escala de Percepción Subjetiva del Esfuerzo creada por Borg. Los valores medios presentaron una diferencia entre la intensidad

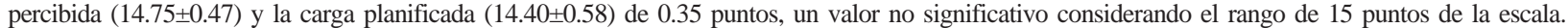
Los resultados obtenidos mostraron una correlación significativa de $\mathrm{r}=0.74$ entre ambas percepciones. Las correlaciones encontradas en los microciclos de mantenimiento de $r=0.87$ y en los descendentes de $r=0.85$ fueron significativas, lo que indica que la intensidad percibida por los jugadores era la que previamente habían planteado los entrenadores. Se ha encontrado una correlación significativa en el método aeróbico ( $\mathrm{r}=0.91$ ), anaeróbico ( $\mathrm{r}=0.83$ ), aláctico ( $\mathrm{r}=0.82)$, mixto $(\mathrm{r}=0.80)$ y específico $(\mathrm{r}=0.63)$. Los resultados muestran que la doble escala permite comprobar y establecer la desviación entre la percepción del jugador y del cuerpo técnico y establecer cómo va asimilando las cargas el jugador posibilitando reajustar la misma según la información recibida.

Palabras clave. Fútbol sala, percepción subjetiva, cargas de entrenamiento, control, doble escala.

Abstract. The purpose of this study is to investigate the relationship between players' perceived intensity and the training load planned by the coaching staff (double scale). Participants 12 professional Spanish football players for an entire season of 40 weeks. For data collection, the Scale of Perceived Exertion created by Borg was used. The mean values showed a difference between the perceived intensity (14.75 \pm 0.47$)$ and the planned load (14.40 \pm 0.58 ) 0.35 points, an non-significant amount considering the 15 points range of the scale . The results showed a significant correlation of $r=0.74$ between the two perceptions. The correlations found in maintenance microcycles, $r=0.87$, and in the downstream ones, $r=0.85$, were significant, indicating that the intensity perceived by players coincided with what coaches had previously planned. We found a significant correlation in the specific aerobic method $(r=0.91)$, anaerobic $(r=0.83)$, alactic $(r=0.82)$, mixed $(r=0.80)$ and specific $(r=0.63)$. The results show that the double scale allows to test and establish the difference between the perception of the player and the coaching staff and establish how the former assimilates loads allowing to adjust then according to the information received.

Keywords. Futsal, subjective perception, training loads, control, double scale.

\section{Introducción}

El control de las cargas de entrenamiento es el medio fundamental en una planificación para conocer el grado de desviación entre el rendimiento y las adaptaciones previstas y las alcanzadas (Seirul-lo, 2008; Balagué \& Torrents, 2006). Mújika (2006) establece que en un plan de entrenamiento existen tres tipos de cargas relacionadas que pueden variar entre sí y deben ser consideradas a la hora de programar los entrenamientos:

- La carga planificada a principios de temporada.

- La carga prescrita el día en que debe llevarse a cabo.

- La carga realizada por el deportista.

Para llevar a cabo una planificación real y ajustada a cada jugador y a cada momento, la implicación del deportista en el proceso de planificación del entrenamiento es necesaria en el entrenamiento actual en los deportes colectivos y la información obtenida del binomio entrenadordeportista será la clave, entre otros objetivos, para prevenir lesiones por falta o exceso de entrenamiento, uno de los motivos principales de lesión en deportes colectivos (Prieto, 2015). El deportista, con su feedback al entrenador, informará de cómo va asimilando las cargas y de las sensaciones que va teniendo, y el entrenador comparará esas informaciones con sus observaciones a lo largo del proceso de entrenamiento y los valores que haya planificado previamente (Casáis, 2008).

La observación directa es un método de cuantificación que permite establecer aspectos del entrenamiento como el tipo, la duración e intensidad de las sesiones y evaluar cuestiones más subjetivas como la impresión global del entrenador con respecto al deportista (Foster, 1998). Este método se basa principalmente en la experiencia del cuerpo técnico y en su conocimiento de los jugadores y el entorno permitiendo dosificar los esfuerzos grupales e individuales. La observación directa elimina cualquier error subjetivo inherente al deportista pero introduce un error subjetivo procedente del observador. Es un método muy válido para la comparación de varios deportistas por parte de un mismo observador, así como para determinar intensidades de entrenamiento siempre que el

Fecha recepción: 09-02-15- Fecha envío revisores: 09-02-15- Fecha de aceptación: 25-06-15 Angeles Filgueira Perez

gelesfp@gmail.com entorno lo permita (Hopkins, 1991).

El uso de la percepción subjetiva del esfuerzo (PSE) ha mostrado ser un método fiable y válido desde sus inicios (Carton \& Rhodes, 1985). Un gran número de trabajos se han ocupado de estudiar si el sistema cumple con dichos criterios en diferentes situaciones, tanto en deportes individuales como colectivos (Coutts, Rampinini, Marcora, Castagna \& Impellizzeri, 2009; Borresen \& Lambert, 2008; Alexiou \& Coutts, 2008). A lo largo de los últimos 40 años se han realizado numerosos estudios que confirman la fiabilidad y validez del método con valores entre r=0,85-0,95 como el de Buckley, Eston \& Sim (2000). Además, es un sistema fácil de aplicar, barato y accesible a prácticamente todos los niveles de entrenamiento tal como establecen Gearhart, Goss, Lagally, Jakicic, Gallagher \& Gallagher (2002).

Se observan correlaciones fuertes entre el método PSE y las otras formas de cuantificación de la carga interna, principalmente la FC, en diferentes estudios en deportes colectivos (Borresen \& Lambert, 2008; Alexiou \& Coutts, 2008), lo que da mayor fiabilidad a esta herramienta subjetiva como medio para la cuantificación del entrenamiento.

En el diseño de la Escala RPE (Borg, 1962), se determinó que para realizar una valoración más completa era preciso utilizar la «doble escala», basada en la percepción del deportista (PSE percibida) confrontada con la previsión del cuerpo técnico (PSE prevista), lo que permite establecer numéricamente la diferencia en la valoración de la carga por parte de ambas partes.

Esta relación entre la valoración de la intensidad de los jugadores con el cuerpo técnico ha sido estudiada tanto en los deportes individuales como atletismo y natación (García \& Leibar, 1997; Fóster, 2001) como en los colectivos: fútbol (Núñez, Carbonel \& Raya, 2004), baloncesto (Peres, Debora \& Del Campo, 2011) y balonmano (Del Campo, 2004). Todos ellos se han realizado en una parte concreta de la temporada y no a lo largo de la misma donde se dan todo tipo de cargas y contenidos de trabajo.

\section{Objetivo}

Comparar y establecer la relación, a través de la percepción subjetiva, de la intensidad del entrenamiento de los jugadores de la carga realizada con la carga planificada por el cuerpo técnico durante toda una temporada en fútbol sala. 


\section{Material y método}

\section{Tipo de estudio}

Se trata de un diseño observacional con medidas repetidas, de tipo longitudinal a lo largo de la temporada 2011/2012 durante 40 semanas en 225 sesiones.

La metodología seguida fue aprobada por el Comité Ético de la Universidad de Zaragoza siguiendo las directrices de la Declaración de Helsinki para investigación con humanos de 1974 y modificada en 2008. Todos los participantes fueron informados del propósito del estudio, se obtuvo su consentimiento y sabían que podían retirarse del mismo cuando consideraran oportuno.

\section{Participantes}

Jugadores de la primera plantilla «Hormigoneras Umacón Zaragoza» de Primera División española de fútbol sala (n=12). Las características de la muestra son: edad; $27.00 \pm 5.12$ años; talla $175.98 \pm 5.94$ cm; peso $73.97 \pm 6.13 \mathrm{~kg}$. La población en la que se incluye la muestra se compone de 14 equipos profesionales.

Los criterios de inclusión fueron; ser jugador de la primera plantilla o del equipo filial y acudir siempre a los entrenamientos, exceptuando causas de lesión o enfermedad puntual. Y los criterios de exclusión; no acudir siempre a los entrenamientos, jugadores con lesiones de larga duración y jugadores que no completaron la mitad de la temporada.

Los 12 sujetos habían participado la temporada anterior en un estudio piloto. La temporalización fue:

- Temporada 2.010/2.011. Estudio piloto

- Agosto a diciembre de 2.010: Se introdujo el método de PSE y la doble escala para constatar si su aplicación diaria era viable.

-Enero a mayo 2.011: Una vez constatada la utilidad de la misma y conseguida la concienciación de su buena utilización por parte de los jugadores se pasó a utilizar sistemáticamente en cada sesión.

- Temporada 2.011/2.012. Implantación total de la herramienta.

\section{Procedimiento de recogida de datos}

Este registro se realizó a través de la Escala RPE (figura 1). Basándonos en los estudios de Nuñez et al. (2004) y Baden, Warwick-Evans \& Lakomy (2004) que demostraron que la consciencia del jugador del tipo de contenido y la intensidad prevista por los entrenadores puede condicionar su percepción, en ningún momento los jugadores eran conscientes de los contenidos a trabajar y de la intensidad prevista. De esta manera se evitaba condicionar sus valoraciones.

\begin{tabular}{|l|l|}
\hline Escala RPE & \\
\hline \multirow{2}{*}{ Muy, muy ligero } & 6 \\
\cline { 2 - 2 } Muy ligero & 7 \\
\hline \multirow{2}{*}{ Ligero } & 8 \\
\hline \multirow{2}{*}{ Algo duro } & 10 \\
\cline { 2 - 2 } Duro & 11 \\
\hline \multirow{2}{*}{ Muy duro } & 12 \\
\hline \multirow{2}{*}{ Muy, muy duro } & 13 \\
\hline & 14 \\
\hline & 16 \\
\cline { 2 - 2 } & 17 \\
\hline & 18 \\
\hline & 20 \\
\hline
\end{tabular}

Los jugadores registraban el valor de PSE percibida en fichas individuales, aislados de los compañeros, al finalizar la sesión justo antes de realizar la parte de los estiramientos en grupo.

Se utilizaron dos fichas:

-Ficha de planificación semanal donde el cuerpo técnico detallaba los contenidos y las cargas de trabajo previstas.

-Ficha de registro diario de PSE percibida por los jugadores y PSE prevista por los entrenadores.

Los tres tipos de microciclos planificados se han clasificado siguiendo a Álvarez et al. (2004), quienes establecen la necesidad de combinar microciclos ascendentes, descendentes y de mantenimiento, teniendo en cuenta que las respuestas adaptativas de los jugadores son distintas y por eso es necesario aplicar distintas cargas según el momento de la temporada para lograr diferentes picos de forma
-Ascendente: aumento de la carga. Orientado al desarrollo de determinadas cualidades físicas.

- Mantenimiento: mantenimiento de la carga. Orientado a la transformación hacia las manifestaciones específicas.

-Descendente: descenso de la carga. Orientado a la recuperación.

Siguiendo a Álvarez et al. (2004), creemos que en los deportes colectivos y debido a la necesidad de tener jugadores resistentes, fuertes y veloces es necesario intercalar situaciones específicas con ejercicios de carácter exclusivamente físico, durante la pretemporada y también en la temporada, en muchas ocasiones mediante un trabajo mixto en el que se combinen ambos tipos de trabajo fundamentalmente, con el objetivo de mejorar las deficiencias individuales y llegar en las mejores condiciones posibles a las situaciones reales de partido. Se han distinguido cinco métodos de entrenamiento, los cuales se trabajan por separado en cada sesión:

- Anaeróbico: trabajo cardiovascular a través de series de carrera cortas e intensas + trabajo de fuerza explosiva.

-Aeróbico: trabajo cardiovascular a través de series de carrera largas y de intensidad media + trabajo de fuerza resistencia.

- Aláctico: situaciones específicas de tiro y velocidad en pista.

-Específico: situaciones de juego similares a la competición.

-Mixto: trabajo cardiovascular en pista (fraccionados o cambios de ritmo) + situaciones de juego.

\section{Método estadístico}

Paquete SPSS versión 19 (Licencia Universidad de Zaragoza) y programa Excel (análisis de datos) para realizar la estadística descriptiva e inferencial de las diferentes variables. En la estadística descriptiva se ha utilizado en el caso de variables cuantitativas, la media y desviación estándar.

- Para obtener las correlaciones y las dependencias lineales entre variables cuantitativas, en el caso de cumplimiento de condición de regresión lineal se ha utilizado la correlación de Pearson (r)

-En todos los casos se han utilizado el nivel habitual de significación $\alpha=.05$. Cuando la correlación es significativa $(\mathrm{p}<.05)$ se señala $=*$ *

\section{Resultados}

Comparación semanal de los valores de PSE percibida y PSE prevista

Los valores medios presentaron una diferencia entre la PSE percibida (14.75 \pm 0.47$)$ y la PSE prevista (14.40 \pm 0.58$)$ de 0.35 no encontrando diferencias significativas. Entendemos que las diferencias encontradas en los resultados fueron mínimas teniendo en cuenta el rango de la escala utilizada del 6 al 20 (tabla 1).

La PSE percibida en 38 semanas estableció una valoración media del equipo de duro (14-15.9) y sólo en dos semanas -semana 10 y 28la valoración fue de algo duro (12-13.9). Respecto a la PSE prevista en 31 semanas la valoración media del cuerpo técnico fue de duro (14-15.9) y en siete semanas -semana $5,10,16,17,19$,28 y 29- la valoración fue de algo duro (12-13.9) (tabla 1).

\begin{tabular}{|c|c|c|c|c|c|c|c|}
\hline Semana & $\begin{array}{c}\text { PSE } \\
\text { percibida }\end{array}$ & $\begin{array}{c}\text { PSE } \\
\text { prevista }\end{array}$ & Diferencia & Semana & $\begin{array}{c}\text { PSE } \\
\text { percibida }\end{array}$ & PSE prevista & Di ferencia \\
\hline 1 & $15,04 \pm 1,30$ & $15,40 \pm 0,97$ & 0,36 & 21 & $14,63 \pm 1,28$ & $14,00 \pm 1,15$ & $-0,63$ \\
\hline 2 & $15,40 \pm 1,45$ & $15,22 \pm 1,64$ & $-0,17$ & 22 & $14,75 \pm 2,00$ & $14,40 \pm \pm 1,14$ & $-0,35$ \\
\hline 3 & $15,16 \pm 1,96$ & $14,44 \pm 1,81$ & $-0,72$ & 23 & $14,66 \pm 0,38$ & $14,25 \pm 0,96$ & $-0,41$ \\
\hline 4 & $15,26 \pm 1,01$ & $14,63 \pm 0,74$ & $-0,64$ & 24 & $15,20 \pm 1,04$ & $14,43 \pm 1,13$ & $-0,77$ \\
\hline 5 & $14,42 \pm 0,37$ & $13,60 \pm 0,89$ & $-0,82$ & 25 & $15,09 \pm 0,67$ & $15,00 \pm 0,82$ & $-0,09$ \\
\hline 6 & $15,36 \pm 0,56$ & $15,50 \pm 1,05$ & 0,14 & 26 & $14,77 \pm 1,05$ & $14,71 \pm 0,95$ & $-0,06$ \\
\hline & $15,05 \pm 1,29$ & $14,71 \pm 2,14$ & $-0,34$ & 27 & $14,25 \pm 1,22$ & $14,17 \pm 1,47$ & $-0,08$ \\
\hline 8 & $15,01+2,48$ & $15,00 \pm 2,28$ & $-0,01$ & 28 & $13,45 \pm 1,67$ & $13,33 \pm 1,21$ & $-0,11$ \\
\hline 9 & $15,18 \pm 1,50$ & $15,00 \pm 1,67$ & $-0,18$ & 29 & $14,09 \pm 1,50$ & $13,60 \pm 1,14$ & $-0,49$ \\
\hline 10 & $13,67 \pm 0,94$ & $13,33 \pm 0,58$ & $-0,33$ & 31 & $14,76 \pm 1,49$ & $15,00 \pm 1,79$ & 0,24 \\
\hline 11 & $14,30 \pm 1,48$ & $14,17 \pm 1,33$ & $-0,13$ & 32 & $15,27 \pm 1,45$ & $15,20 \pm 1,64$ & $-0,07$ \\
\hline 12 & $14,57 \pm 1,10$ & $14,67 \pm 1,51$ & 0,09 & 33 & $14,44 \pm 1,37$ & $14,20 \pm 1,30$ & $-0,24$ \\
\hline 13 & $14,05 \pm 2,81$ & $14,00 \pm 2,28$ & $-0,05$ & 34 & $14,80 \pm 1,35$ & $14,40 \pm 1,52$ & $-0,40$ \\
\hline 14 & $15,20 \pm 1,08$ & $14,40 \pm 1,34$ & $-0,80$ & 35 & $14,55 \pm 1,90$ & $14,00 \pm 2,35$ & $-0,55$ \\
\hline 15 & $14,87 \pm 1,59$ & $14,80 \pm 1,79$ & $-0,07$ & 36 & $14,75 \pm 2,55$ & $14,20 \pm 1,92$ & $-0,55$ \\
\hline 16 & $14,49 \pm 2,41$ & $13,83 \pm 1,72$ & $-0,65$ & 37 & $14,91 \pm 1,58$ & $14,00 \pm 0,71$ & $-0,91$ \\
\hline 17 & $14,01 \pm 1,73$ & $13,50 \pm 1,64$ & $-0,51$ & 38 & $15,16 \pm 1,38$ & $14,50 \pm 1,00$ & $-0,66$ \\
\hline 19 & $15,09 \pm 0,99$ & $13,60 \pm 1,82$ & $-1,49$ & 39 & $14,85 \pm 2,37$ & $14,50 \pm 1,91$ & $-0,35$ \\
\hline 20 & $14,84 \pm 1,50$ & $15,25 \pm 0,96$ & 0,41 & 40 & $15,00+1,68$ & $14,33 \pm 2,25$ & $-0,67$ \\
\hline MEDIA & $14,75 \pm 0,47$ & $14,40 \pm 0,58$ & $-0,35$ & $\mathrm{R}$ & & $0,74^{*}$ & \\
\hline
\end{tabular}


Los resultados obtenidos mostraron una correlación significativa de r=0.74* entre la PSE percibida y la PSE prevista del equipo.

\section{Tendencia semanal}

La evolución de la PSE a lo largo de la semana indica la intensidad media de la carga interna que estaban soportando nuestros deportistas cada día. Secomprueba como a lolargo de la semana ambas PSE variaban, siendo la PSE percibida mayor en todas las sesiones excepto el LM y el JT (tabla 2).

Tabla 2.

Percepcín subjetiva del esfuerzo del depotista y previsín de la ine

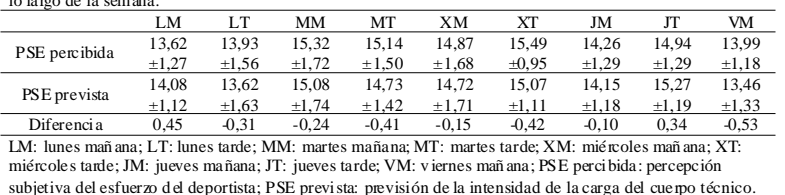

\section{Comparación según los tipos de microciclos}

En los doce microciclos ascendentes se comprueba como en nueve de ellos la PSE percibida fue mayor que la PSE prevista y sólo en tres microciclo 1, 5 y 10- ocurrió lo contrario. En los 19 microciclos de mantenimiento se obtuvo como en 17 de ellos la PSE percibida fue mayor que la prevista y sólo en dos microciclos -microciclo 4 y 9ocurrió lo contrario. En los sietemicrociclos descendentes la PSE percibida fue mayor que la prevista (figura 2).

Las diferencias entre la PSE percibida y prevista entre los tipos de microciclo oscilaron entre 0.25 y 0.58 . Se observa como la correlación de la doble escala son significativas en los microciclos de mantenimiento y descendentes, y no lo es en los ascendentes (tabla 3).
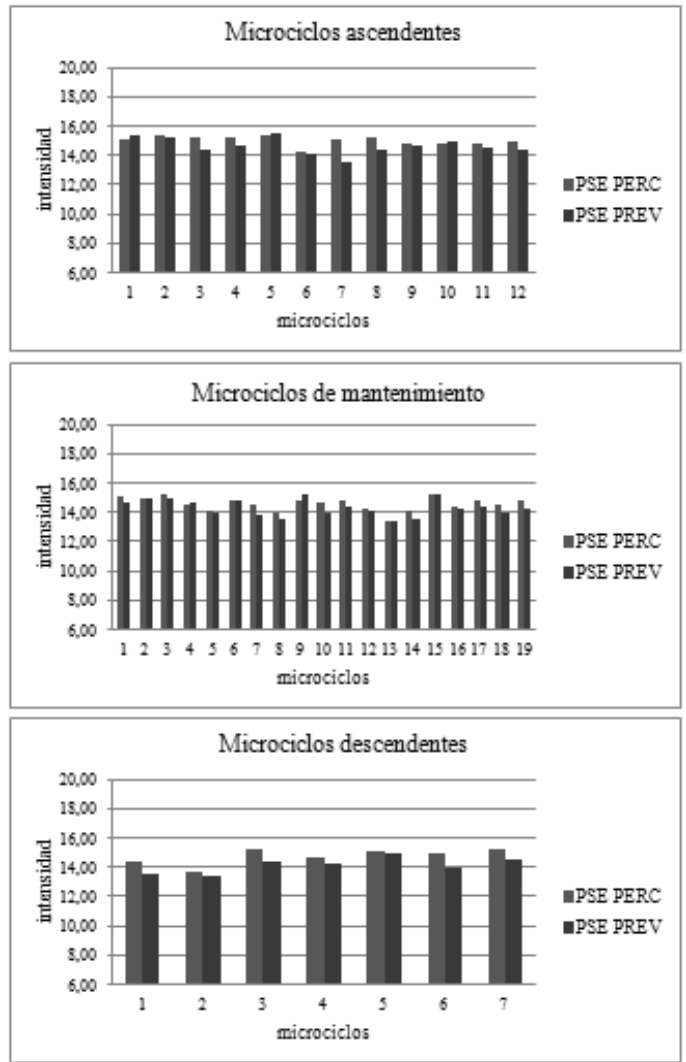

Figura 2. Diferencia de la percepción subjetiva del esfuerzo del deportista y la previsión de la intensidad de la carga del cuerpo técinico según el tipo de microciclo.

PSE PERC: percepción subjetiva del esfuerzo del deportista; PSE PR EV: previsión de la inten sidad de la carga del cuerpo técnico.

Tabla 3

Valor medio de la percepción subjetiva del esfuerzo del deportista y la previsión de la in tensidad de la carga del cuer po técnico seqún tipo de microciclo.

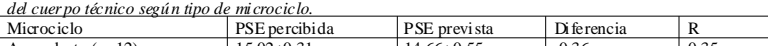
Microciclo \begin{tabular}{lll}
\hline Mantenimiento $(\mathrm{n}=19)$ & $14,58 \pm 0,46$ \\
\hline Desc & &
\end{tabular} \begin{tabular}{l|l|l|l|l}
\hline & $14,73 \pm 0,55$ & $14,15 \pm 0,56$ & $-0,58$ & $0,85^{*}$
\end{tabular} PSE perabi da: percepción subjetiva del carga del cuepo o técnico; R: correlación.
Comparación según los tipos de métodos de entrenamiento

El método aeróbico fue el que presentó un valor menor en ambas PSE.

El resto de métodos de entrenamiento presentaron valores en ambas PSE superiores a 14 puntos. El método mixto presentó los valores más altos, en la PSE percibida de $16.08 \pm 1.03$ y en la PSE prevista de $16.06 \pm 1.03$ (tabla 4).

Los resultados mostraron correlación significativa de la doble escala en el método aeróbico ( $\mathrm{r}=0.91^{*}$ ), anaeróbico $\left(\mathrm{r}=0.83^{*}\right)$, aláctico $(\mathrm{r}=0.82 *)$, mixto $\left(\mathrm{r}=0.80^{*}\right)$ y específico $\left(\mathrm{r}=0.63^{*}\right)$.

Tabla 4.

Comparación de la percepción subjetiva del esfuerzo del deporti sta y la previsión de la intensidad de la carga del cuerpo técnico según los métodos de entrenamiento.

\begin{tabular}{|c|c|c|c|c|}
\hline & PSE percibida & PSE prevista & Diferencia & $\mathrm{R}$ \\
\hline Anaeróbico $(\mathrm{n}=23)$ & $15,48 \pm 0,95$ & $15,60 \pm 0,55$ & 0,12 & $0,83^{*}$ \\
\hline Aeró bico $(\mathrm{n}=25)$ & $12,72 \pm 2,36$ & $13,08 \pm 2,14$ & 0,36 & $0,91^{*}$ \\
\hline Aláctico $(\mathrm{n}=23)$ & $14,99 \pm 1,02$ & $14,75 \pm 1,13$ & $-0,24$ & $0,82^{*}$ \\
\hline Específico $(\mathrm{n}=98)$ & $15,07 \pm 1,02$ & $14,71 \pm 1,15$ & $-0,36$ & $0,63^{*}$ \\
\hline Mixto $(\mathrm{n}=56)$ & $16,08 \pm 1,03$ & $16,06 \pm 1,03$ & $-0,02$ & $0,80^{*}$ \\
\hline
\end{tabular}

\section{Discusión}

Comparación semanal de los valores de PSE percibida y PSE prevista

Estos resultados permitirán comprobar globalmente las diferencias semanales de toda la temporada y establecer la tendencia en la valoración de las cargas por parte de los jugadores y del cuerpo técnico.

Los resultados presentados en la tabla 1 son similares a los que muestra Del Campo (2004) en su estudio con 10 equipos de baloncesto durante ocho semanas, en el que determina en entrenamientos técnicotácticos una PSE prevista por el entrenador de 14 y percibida por los jugadores de 14, encontrándose valores entre seis y 18 y en entrenamientos físicos una PSE prevista por el preparador físico de $13 \mathrm{y}$ percibida por los jugadores de 13, encontrándose valores entre 6 y 18 . Los resultados encontrados por Peres et al. (2011) en un equipo de baloncesto de 11 jugadores durante ocho semanas muestran valoraciones inferiores pero con una diferencia muy baja entre la PSE prevista por el entrenador de 12.37 y la PSE percibida por los jugadores de 12.71. En el estudio de Cuadrado (2010) con 13 jugadores profesionales de balonmano durante 21 sesiones obtiene una PSE percibida de 16.42 en un rango entre 14.36 y 17.82. La PSE prevista por el cuerpo técnico fue de 15.80 en un rango entre 14.50 y 17.00 .

Las diferencias tan bajas encontradas en nuestro estudio y en los estudios presentados demuestran que los deportistas trabajaron a la intensidad requerida por los entrenadores, lo cual indica quela cuantificación a través de la observación directa realizada por sujetos experimentados ha sido en estos casos un buen indicador de la carga realizada como ya indicaba Foster (1998). Para utilizar la doble escala como parámetro de control es necesario tener un conocimiento profundo de la materia por parte del cuerpo técnico y una adecuada percepción del esfuerzo por parte de los jugadores que permita que cada vez la percepción del entrenador y de los jugadores sea más ajustada.

Otros estudios han tratado de comparar las impresiones de jugadores y entrenadores pero sin utilizar la escala de PSE. En el estudio de García-Mas, Agudo, Cuartero, Calabria, Jiménez \& Pérez (2003) con 85 jóvenes de disciplinas individuales y sus respectivos entrenadores comprueban que la percepción del cansancio subjetiva de los deportistas difiere claramente de la que llevan a cabo sus entrenadores. Éstos describen a sus deportistas como más descansados, y es de suponer que actúan en consecuencia en la programación de los entrenamientos y de la competición. Los entrenadores ven cansados a sus deportistas un 17.3\% del tiempo, sin embargo los deportistas se sienten cansados un $63.5 \%$, siendo la diferencia entre la percepción de entrenadores y jugadores muy alta.

Nuestros resultados coinciden con Del Campo (2004) y Cuadrado (2010), se observa que los jugadores percibían por encima de lo previsto por el cuerpo técnico, concretamente en 33 semanas (86.84\%). Estos datos reflejaban que el cuerpo técnico planificaba generalmente una 
carga de trabajo con una intensidad superior a la que percibían los jugadores.

Por otro lado, es importante explicar las diferencias encontradas en el microciclo 19 en el que la PSE percibida fue 1.49 puntos por encima de la prevista. Como entrenadores interesaba conocer los motivos que causaron esa discordancia en la doble escala. El microciclo 19 era singular debido a que en él no había competición y tan sólo se entreno tres días, una sesión el lunes por la tarde y doblando entrenamientos martes y miércoles, es decir, cinco sesiones en tan sólo tres días, con unos contenidos de trabajo exigentes (resistencia aeróbica, fuerza resistencia, cambios de ritmo, situaciones reducidas de juego y partidos). La PSE percibida por el equipo fue $15.09 \pm 0.99$ similar a la mostrada en semanas normales, sin embargo la PSE prevista por el cuerpo técnico fue 13.60 1.82 , muy inferior a la habitual. En este sentido, se considera que el cuerpo técnico valoró la frecuencia de las sesiones antes que la intensidad del trabajo, considerando que al ser una semana en la que tan sólo se entrenaba tres días, los jugadores no percibirían las sesiones tan duras como posteriormente hicieron.

Los resultados obtenidos mostraron una correlación significativa de r=0.74* entre la PSE percibida y la PSE prevista del equipo, igual a la encontrada por Foster, Heimann, Esten, Brice \& Porcari (2001) en su estudio con atletas, el cual obtiene una r $=0.75$ y ligeramente por debajo de la encontrada por Cuadrado (2010) en su estudio con jugadores de balonmano $r=0.80$. Hay que aclarar que en el primer estudio se registraron cinco semanas de entrenamiento y en el segundo estudio se registraron 21 sesiones frente a las 40 semanas y 225 sesiones de nuestro estudio.

Nuestros resultados adquieren mayor importancia si se tiene en cuenta que se obtuvieron durante una temporada completa donde se realizaron entrenamientos con contenidos e intensidades muy distintas y no solo de un mesociclo de la temporada con un objetivo concreto.

\section{Tendencia semanal}

Estos datos permitirán establecer la evolución de la valoración de la carga de jugadores y entrenadores a lo largo del microciclo.

A partir de los resultados obtenidos en la tabla 2 diariamente se podía comprobar si la intensidad percibida por los jugadores había sido mayor o menor a la media y a la previsión del cuerpo técnico, lo que permitía valorar las diferencias encontradas tanto individual como colectivamente.

La comparación de ambas PSE a lo largo de la semana y la utilización de la doble escala permitióllevar a cabo una continua reflexión sobre el esfuerzo real que sufrían los deportistas diariamente y evitar de esta forma la falta de sensibilidad que presentan las planificaciones tradicionales en las que no se tienen en cuenta las sensaciones reales de los jugadores tal como indica Mújika (2006), el cual considera fundamental controlar la carga real y no sólo la carga planificada, ya que lo lógico es que aparezcan diferencias entre ambas.

\section{Comparación según los tipos de microciclos}

Los resultados obtenidos mostrarán la discordancia en la doble escala en los diferentes tipos de microciclos teniendo en cuenta que el volumen y la intensidad de las cargas serán distintas.

Los resultados presentados en la figura 2 demuestran que el cuerpo técnico, independientemente del tipo de microciclo, fue capaz de controlar y conseguir los efectos planificados en los deportistas, algo muy importante en el fútbol sala teniendo en cuenta tal y como establecen Lorenzo \& Mundina (2001) que en los deportes colectivos se deben variar los contenidos en función del estado de los jugadores, los partidos de competición y el momento de la temporada, lo que hace que determinados métodos y contenidos de entrenamiento favorezcan más a unos jugadores que a otros debido a la heterogeneidad de perfiles de un equipo, lo cual debe ser controlado por el cuerpo técnico.

Las correlaciones obtenidas en la tabla 3 en función de los tipos de microciclos determinaron que en los microciclos ascendentes la correlación no fue significativa obteniéndose una r $=0.35$. Esto pudo ser debido a que en los microciclos ascendentes fue donde se aplicaban las cargas de entrenamiento de intensidades más elevadas, principalmente entre el 80-90\% de la capacidad máxima de los deportistas, por lo que las respuestas de los jugadores fueron muy variadas debido a sus diferencias intersujetos. Estos resultados coinciden con los obtenidos por Little \& Williams (2007) que determinan una menor validez del método PSE a intensidades altas de trabajo. Y al mismo tiempo son contrarios a los obtenidos en otros estudios (Borg, 1962; Carton \& Rhodes, 1985; Eston \& Williams, 1998; Yamaji, Yakata \& Shephard, 1992) que establecen una mayor validez cuando las cargas son altas. En base a estos resultados es necesario acortar la discordancia entre ambas valoraciones ya que en el caso de no ser corregida puede ocurrir que los contenidos y las cargas planteadas en este tipo de microciclos ascendentes no tengan los efectos esperados por los entrenadores, o lo tengan para unos pero no para otros jugadores.

Tal como expone Terrados, Mora \& Padilla (2004) para poder corregir estas discrepancias la forma correcta sería hacer las valoraciones previstas de forma individual para cada deportista teniendo en cuenta que en función de sus características condicionales, fisiológicas, psicológicas, técnicas y de experiencia deportiva, cada jugador tienen un ritmo propio de asimilación de las cargas de entrenamiento que hace que cada tipo de contenido le afecte de forma distinta que a sus compañeros. Esto convierte al entrenador tal como lo define Jones (2006) en un auténtico gestor de las necesidades de sus deportistas.

Las correlaciones encontradas en los microciclos de mantenimiento de $r=0.87 *$ y en los descendentes de $r=0.85 *$ si fueron significativas, lo que hizo que la intensidad percibida por los jugadores fuera la que previamente habían planteado los entrenadores.

\section{Comparación según los tipos de métodos de entrenamiento}

Estos datos permitirán determinar las diferencias de la doble escala en diferentes contenidos de trabajo.

Las sesiones de tipo aeróbico, como muestra los resultados (tabla 4) fueron efectivamente las que menos intensidad debían suponer para los jugadores, al tratarse de contenidos que pretendían recuperar al organismo de esfuerzos muy intensos y prepararle para soportar las siguientes cargas de entrenamiento de forma óptima. Estos resultados en las sesiones de baja intensidad son superiores a los mostrados en el estudio llevado a cabo por Foster et al. (2001) con 15 corredores a lo largo de un período de cinco semanas a través de la Escala CR-10 de 10 puntos (Borg, 1982). En las sesiones consideradas de intensidad baja, los atletas percibieron un PSE de 2.4 que corresponde a valores de ocho o nueve en la Escala de RPE según Buceta (1998) y los entrenadores plantearon una PSE de 1.8 que corresponde a valores de siete.

Sin embargo, los valores del resto de los métodos, no coinciden con la PSE percibida encontrada en el estudio de Foster ya que en las sesiones de entrenamiento consideradas de alta intensidad, los jugadores percibieron un PSE de 6.2 en la Escala CR-10 y de 14 en la Escala de RPE. Sin embargo, sí coinciden con la PSE prevista por el cuerpo técnico, con una valor de 7.1 en la escala CR-10 y de 15-16 en la Escala deRPE.

Los resultados obtenidos permite confirmar que, independientemente del tipo de contenido de entrenamiento, los valores de la doble escala fueron muy similares apreciándose diferencias mínimas en todos los métodos, lo que asegura que el equipo trabajó siempre a la intensidad planteada por el cuerpo técnico evitando una mala adaptación al esfuerzo y garantizando la mejora del rendimiento al contrario de la conclusión a que llega Foster et al. (2001), donde la no correspondencia entre el programa de entrenamiento diseñado por los entrenadores y el realizado por sus deportistas es el responsable de muchos fracasos competitivos y debe ser analizado para encontrar posibles soluciones y evitar que vuelva a suceder.

Las correlaciones encontradas (tabla 4) permiten garantizar que el equipo en todos los tipos de sesiones trabajó a la intensidad prevista.

\section{Conclusiones}

-La previsión de la intensidad de la carga planificada por parte del 
cuerpo técnico con respecto a la percepción subjetiva de la intensidad por parte de los jugadores permite comprobar y establecer la desviación entre ambas.

-La doble escala permite establecer cómo va asimilando las cargas el jugador y da la posibilidad de reajustar la misma según la información recibida.

\section{Implicaciones teórico-prácticas futuras}

-La utilización de métodos invasivos para el control de las cargas de entrenamiento como la monitorización de la frecuencia cardiaca, el control del consumo máximo de oxígeno o la medición del lactato sanguíneo no aportan datos inmediatos para comparar las respuestas de los deportistas con la planificación de los entrenadores.

- La respuesta de cada deportista en cada método de entrenamiento depende de sus características individuales en función de aspectos psicofisiológicos muy difíciles de cuantificar, que tratan de relacionar la respuesta fisiológica del sujeto con su conducta. Para ello la percepción subjetiva aporta una información útil a los entrenadores sobre el estado real de sus jugadores.

\section{Referencias bibliográficas}

Alvarez, J., Manonelles, P., Corona, P. (2004.) Planificación y cuantificación del entrenamiento en una temporada regular de fútbol sala. Apunts Educación Física y Deportes, 76, 58-62.

Alexiou, H. \& Coutts, A.J. (2008). A comparison of methods used for quantifying internal training load in women soccer players. Int $J$ Sports Psychiol Perform, 3 (3), 320-330.

Baden, D., Warwick-Evans, L. \& Lakomy, J. (2004). Am I nearly there? The effect of anticipated running distance on perceived exertion and attentional focus. Journal of Sports and Exercise Psychology, 26, 12-19.

Balagué, N. \& Torrents, C. (2006). Adaptación al entrenamiento: de los mecanismos de regulación y control a la autoorganización. RED, 20 (3), 11-18.

Borg, G. (1962). A simple rating scale for use in physical work test. Fysiografiska Sdllskapets y Lund Forhandlingar, 32, 7-15.

Borg, G. (1982). A category scale with ratio properties for intermodal and interindividual comparisons. En: Geissler, H.G, Petzold, P., editores. Psychophysical judgment and the process of perception. Deutscher Verlag der Wissenschaften, 25-34.

7. Borresen, J. \& Lambert, M.I. (2008). Quantifying training load: A comparison of subjective and objective methods. Int J Sports Psychiol Perform, 3 (1), 16-30.

Buceta, J.M. (1998). Psicología del entrenamiento deportivo. Madrid: Dykinson.

Buckley, J., Eston, R. \& Sim, J. (2000). Ratíngs of perceíved exertion in braile: validity and reliability in production mode, Sports Med, 34 (4), 297-302.

Carton, R.L. \& Rhodes, E.C. (1985). A critical review of the literature on ratings scales for perceived exertion. Sports Medicine, 2, 198222.

Coutts, J., Rampíníní, E., Marcora, S.M., Castagna, C. Impellízzeri, F.M. (2009). Heart rate and blood lactate correlates of perceived exertion during small-sided soccer games. J Sci Med Sport, 12(1), 79-84.

Casais, L. (2008). Revisión de las estrategias para la prevención de lesiones en el deporte desde la actividad física. Apunts, 157, 30-40.

Cuadrado, J. (2010). Análisis de la influencia de la intensidad del entrenamiento sobre variables de control de la carga interna en deportes colectivos. [Tesis Doctoral]. Facultad de ciencias de la actividad física y el deporte. Universidad de Granada.

Del Campo, J. (2004). La intensidad del entrenamiento en jugadores de baloncesto medida a través de la percepción de esfuerzo y fatiga [Tesis doctoral]. Departamento de Físicas e Instalaciones Aplicadas a la Edificación, al Medio Ambiente y al Urbanismo. E.T.S.
Arquitectura. Universidad de Madrid.

Eston, R. \& Williams, J.G (1998). Reliability of rating of perceiver effort regulation of exercise intensity. British Journal of Sports Medicine, 22 (4), 153-155.

Foster, C. (1998). Monitoring training in athletes with reference to overtraining syndrome. Medicine and Science in Sports and Exercise, 30, 1164-1168.

Foster, C., Heimann, K.M., Esten, P.L., Brice, G \& Porcari, J.P. (2001). Differences in Perceptions of Training by Coaches and Athletes. Sports Medicine, 8, 3-7.

García, M. \& Leibar, X. (1997). Entrenamiento de la resistencia de los corredores de medio fondo y fondo. Madrid: Gymnos.

García-Mas, A., Aguado, F.J., Cuartero, J., Calabria, E., Jiménez, R. \& Pérez, P. (2003). Sueño, descanso y rendimiento en jóvenes deportistas de competición. Revista de Psicología del Deporte, 12 (2), 181-195.

Gearhart, R., Goss, F.L., Lagally, K., Jakicic, J., Gallagher, J. \& Gallagher, K. (2002). Ratings of perceived exertion in active muscle during high-intensity and low-intensity resistance exercise. JStrength Cond Res, 16 (1), 87-91.

Hopkins, WG. (1991). Quantification of training in competitive sports, methods and applications. Sports Medicine, 12 (3), 161-183.

Jones, R. (2006). The Sports coach as educator: re-conceptualising sports coaching. London: Routledge.

Little, T. \& Williams, A.G. (2007). Measures of exercise intensity during soccer training drills with proiessional soccer piayers. Journal of Strength and Conditional Research, 21 (2), 367-371.

Lorenzo, A. \& Mundina, J. (2001). Los principios del entrenamiento deportivo. Malaga: Curso de entrenador de segundo nivel de baloncesto. Preparación Física. FEB.

Mujika, I. (2006). Métodos de cuantificación de las cargas de entrenamiento y competición. Kronos: revista universitaria del la actividad física y el deporte, 10, 45-54.

Nuñez, F., Carbonel, A. \& Raya, A. (2004). Valoración subjetiva del esfuerzo aplicada al entrenamiento específico en fútbol. EFdeportes, 73, 1-4. Recuperado de http://www.efdeportes.com/efd73/subj.htm

Peres. J., Debora,A. \& Del Campo, J. (2011). Evaluación de la intensidad del entrenamiento de baloncesto a través del salto vertical y RPE. EFdeportes, 152, 1-2. Recuperado de http:// www.efdeportes.com/efd152/la-intensidad-del-entrenamiento-debaloncesto.htm

Prieto, JA. (2015). Variables deportivas y personales en la ocurrencia de lesiones deportivas. Diferencias entre deportes individuales y colectivos. Retos, 28, 21-25.

Seirul-lo, F. (2008). Master Profesional en Alto Rendimiento Deportivo Deportes de Equipo. Notas de clase del Módulo: «Planificación del entrenamiento en los deportes de equipo». Barcelona: Byomedic y Fundación F. C. Barcelona.

Terrados, N., Mora, R. \& Padilla, S. (2004). La Recuperación de la fatiga del deportista. Madrid: Gymnos.

Yamaji, K., Yakata, Y. \& Shephard, R.J. (1992). A comparison of the perceived and the ECG measured heart rate during cycle erqometer, treadmill and stairmill exercise before and after perceived heart rate training. Journal of Sports Medicine and Physical Fitness, 34, 271281.

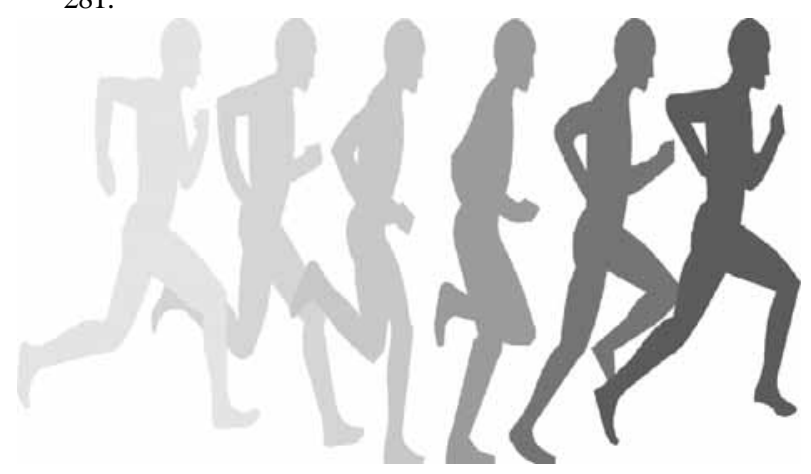

